\title{
The MUonE experiment
}

\author{
Umberto Marconi ${ }^{1, *}$ \\ ${ }^{1}$ Via Irnerio, 4640126 Bologna, Italy
}

\begin{abstract}
The precision measurement of the anomalous magnetic moment $g-2$ of the muon at present exhibits a $3.5 \sigma$ deviation between theory and experiments. In the next few years it will be measured to higher precisions at Fermilab and J-PARC. The theoretical prediction can be improved by reducing the uncertainty on the leading hadronic correction $a_{\mu}^{H L O}$ to the $g-2$. Here we present a new approach to determine $a_{\mu}^{H L O}$ with space-like data, by means of a precise measurement of the hadronic contribution to the effective electromagnetic coupling $\alpha$, exploiting the elastic scattering of $150 \mathrm{GeV}$ muons (currently available at CERN North area) on atomic electrons of a low-Z target. The direct measurement of $a_{\mu}^{H L O}$ in the space-like region will provide a new independent determination and will consolidate the theoretical prediction of the muon $g-2$ in the Standard Model. It will allow therefore a firmer interpretation of the measurements of the future muon $g-2$ experiments at Fermilab and J-PARC.
\end{abstract}

\section{Introduction}

The discrepancy between the experimental value of the muon anomalous magnetic moment $a_{\mu}=(g-2) / 2$ and the Standard Model (SM) prediction, $\Delta a_{\mu} \sim(28 \pm 8) \times 10^{-10}$ is a long standing issue in particle physics $[1,2]$. The current accuracy of the SM predictions, $\sim 5 \times 10^{-10}$, are limited by strong interaction effects, at the low energy scale implied. However by using analyticity and unitarity, it was shown [3] that the leading-order (LO) hadronic contribution to the muon $g-2, a_{\mu}^{\mathrm{HLO}}$, can be computed via a dispersion integral of the hadron production cross section in $e^{+} e^{-}$annihilation at low-energy. The present error on $a_{\mu}^{\mathrm{HLO}}, \sim 4 \times 10^{-10}$ or slightly better, with a relative accuracy of $0.6 \%$, constitutes the main uncertainty of the SM prediction [2]. Alternative evaluations of $a_{\mu}^{\mathrm{HLO}}$ can be obtained with QCD lattice calculations [4]. The current lattice QCD results are not yet competitive with the dispersive approach via time-like data, with errors that are expected to decrease significantly in the next few years [5]. The $O\left(\alpha^{3}\right)$ hadronic light-by-light contribution, $a_{\mu}^{\mathrm{HLbL}}$, which has the second largest error in the theoretical evaluation, contributing with an uncertainty of (2.5$4) \times 10^{-10}$, cannot at present be determined from data and its calculation relies on the use of specific models [6-8].

The error achieved by the BNL E821 experiment [9], $\delta a_{\mu}^{\operatorname{Exp}}=6.3 \times 10^{-10}$, corresponding to $0.54 \mathrm{ppm}$, is dominated by the available statistics. New experiments at Fermilab and JPARC, aiming at measuring the muon $g$-2 to a precision of $1.6 \times 10^{-10}(0.14 \mathrm{ppm})$, are in preparation $[10,11]$.

\footnotetext{
*e-mail: umberto.marconi@bo.infn.it
} 
Together with the experimental plans, an improvement on the value of the LO hadronic contribution is highly desirable. The proposal described here is to determine $a_{\mu}^{\mathrm{HLO}}$ from a measurement of the effective electromagnetic coupling $\alpha$ in the space-like region, where the vacuum polarization is expectd to be a smooth function of the squared momentum transfer. In this approach the hadronic contribution to the running of $\alpha$ can be measured by means of the $t$-channel $\mu-e$ elastic scattering process, from which $a_{\mu}^{\mathrm{HLO}}$ can be determined directly [12] ${ }^{1}$.

\section{Measuring the Hadronic Leading contribution with space-like data.}

For the calculation of the hadronic leading contribution $a_{\mu}^{\mathrm{HLO}}$ with the $t$-channel approach an alternative formula to the dispersion integral $[3,16]$ can be exploited $[13,17]$, namely:

$$
a_{\mu}^{\mathrm{HLO}}=\frac{\alpha}{\pi} \int_{0}^{1} d x(1-x) \Delta \alpha_{\mathrm{had}}[t(x)],
$$

where $\Delta \alpha_{\text {had }}(t)$ is the hadronic contribution to the running of the fine-structure constant, evaluated at

$$
t(x)=\frac{x^{2} m_{\mu}^{2}}{x-1}<0
$$

that is the space-like (negative) squared four-momentum transfer of the process. In contrast with the dispersive integral, the integrand of Eq. (1) is a smooth functin and free of resonance poles. Fig. 1 (left) shows $\Delta \alpha_{\text {had }}$, and for comparison $\Delta \alpha_{\text {lep }}$, as a function of the variables $x$ and $t$. The range $x \in(0,1)$ corresponds to $t \in(-\infty, 0)$, with $x=0$ for $t=0$. The expected integrand of Eq. (1), calculated with the routine hadr $5 \mathrm{n} 12$ [18], which uses timelike hadroproduction data and perturbative QCD, is plotted in Fig. 1 (right). The peak of the integrand occurs at $x_{\text {peak }} \simeq 0.914$ (corresponding to $\left.t_{\text {peak }} \simeq-0.108 \mathrm{GeV}^{2}\right)$ and $\Delta \alpha_{\text {had }}\left(t_{\text {peak }}\right) \simeq$ $7.86 \times 10^{-4}$ (see Fig. 1 (right)) .

We propose to use Eq. (1) to determine $a_{\mu}^{\mathrm{HLO}}$ by measuring the running of $\alpha$ using the CERN muon beam of energy $E_{\mu}=150 \mathrm{GeV}$, colliding on electron at rest of a fixed target. This technique is similar to the one used for the measurement of the pion form factor, as described in [19].

It looks very appealing for the following reasons:

- It is a $t$-channel process, making the dependence on $t$ of the differential cross section proportional to $|\alpha(t) / \alpha(0)|^{2}$ :

$$
\frac{d \sigma}{d t}=\frac{d \sigma_{0}}{d t}\left|\frac{\alpha(t)}{\alpha(0)}\right|^{2},
$$

where $d \sigma_{0} / d t$ is the effective Born cross section, including virtual and soft photons, analogously to Ref. [20], where small-angle Bhabha scattering at high energy was considered. The vacuum polarization effect, in the leading photon $t$-channel exchange, is incorporated in the running of $\alpha$ and gives rise to the factor $|\alpha(t) / \alpha(0)|^{2}$.

- Given the incoming muon energy $E_{\mu}$ the $t$ variable is related to the energy of the scattered electron $E_{e}^{\prime}$ or its angle $\theta_{e}$ through:

$$
\begin{gathered}
t=\left(p_{\mu}-p_{\mu}^{\prime}\right)^{2}=\left(p_{e}-p_{e}^{\prime}\right)^{2}=2 m_{e}^{2}-2 m_{e} E_{e}^{\prime}, \\
s=\left(p_{\mu}^{\prime}+p_{e}^{\prime}\right)^{2}=\left(p_{\mu}+p_{e}\right)^{2}=m_{\mu}^{2}+m_{e}^{2}+2 m_{e} E_{\mu},
\end{gathered}
$$

\footnotetext{
${ }^{1}$ The method has been originally proposed [13] by using Bhabha scattering data. A method to determine the running of $\alpha$ by using small-angle Bhabha scattering was proposed in [14] and applied to LEP data in [15].
} 

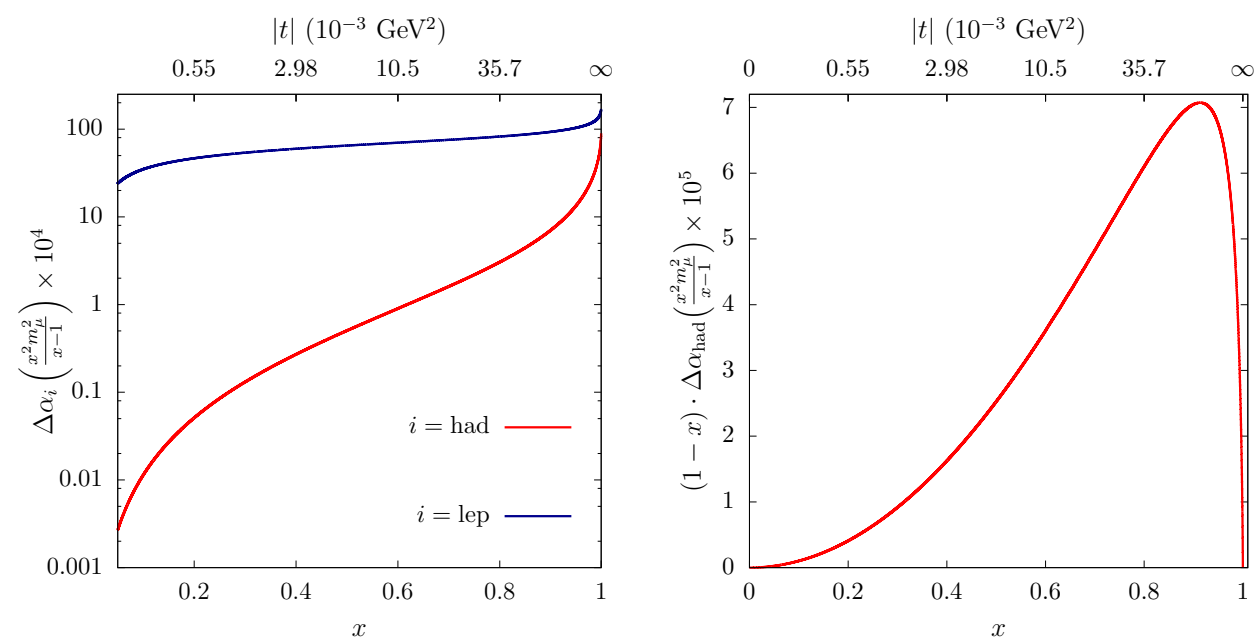

Figure 1. Left: $\Delta \alpha_{\text {had }}[t(x)] \times 10^{4}$ (red) and, for comparison, $\Delta \alpha_{\text {lep }}[t(x)] \times 10^{4}$ (blue), as a function of $x$ and $t$ (upper scale). Right: the integrand $(1-x) \Delta \alpha_{\text {had }}[t(x)] \times 10^{5}$ as a function of $x$ and $t$. The peak value is at $x_{\text {peak }} \simeq 0.914$, corresponding to $t_{\text {peak }} \simeq-0.108 \mathrm{GeV}^{2}$.

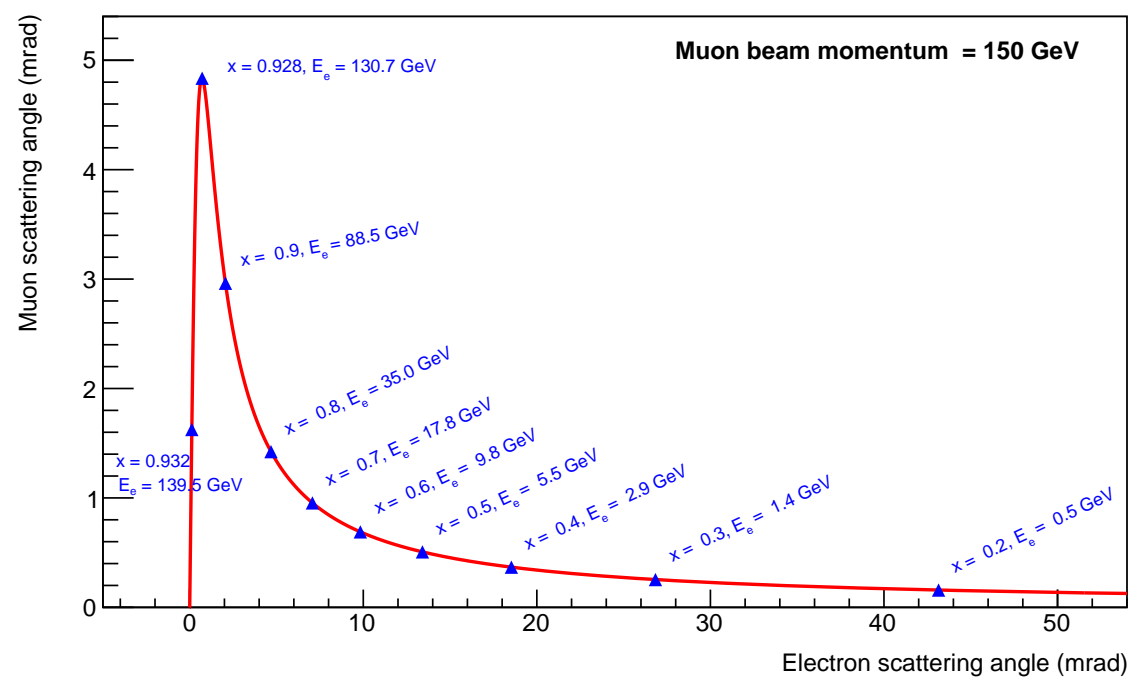

Figure 2. The relation between the muon and electron scattering angles for $150 \mathrm{GeV}$ incident muon beam momentum. Blue triangles indicate reference values of the Feynman's $x$ and electron energy.

$$
E_{e}^{\prime}=m_{e} \frac{1+r^{2} \cos ^{2} \theta_{e}}{1-r^{2} \cos ^{2} \theta_{e}}, \quad \theta_{e}=\arccos \left(\frac{1}{r} \sqrt{\frac{E_{e}^{\prime}-m_{e}}{E_{e}^{\prime}+m_{e}}}\right),
$$

where the angle $\theta_{e}$ spans the range (0-31.85) mrad for the electron energy $E_{e}^{\prime}$ in the range (1-139.8) GeV. 
- For $E_{\mu}=150 \mathrm{GeV}$, it turns out that $s \simeq 0.164 \mathrm{GeV}^{2}$ and $-0.143 \mathrm{GeV}^{2}<t<0 \mathrm{GeV}^{2}$. It implies that the region of $x$ extends up to 0.93, while the peak of the integrand function of Eq. (1) is at $x_{\text {peak }}=0.914$, corresponding to an electron scattering angle of $1.5 \mathrm{mrad}$, as visible in Fig. 1 (right).

- The angles of the scattered electron and muon are correlated as shown in the Fig. 2, drawn for incoming muon energy of $150 \mathrm{GeV}$. This constraint is extremely important to select elastic scattering events, rejecting background events from radiative or inelastic processes and to minimize systematic effects in the determination of $t$. Note that for scattering angles of (2-3) mrad there is an ambiguity between the outgoing electron and muon, as their angles and momenta are similar, to be resolved by means of $\mu / e$ discrimination.

- The boosted kinematics allows the same detector to cover the whole acceptance. Many systematic errors, e.g. on the efficiency, will cancel out (at least at first order) in the relative ratios of event counts in the high and low $q^{2}$ regions (signal and normalization regions).

Assuming to use a muon beam of $150 \mathrm{GeV}$ with an average intensity of $\sim 1.3 \times$ $10^{7} \mathrm{muon} / \mathrm{s}$, with a running time of $2 \times 10^{7} \mathrm{~s} / \mathrm{yr}$, and using 30 experimental points in $x$ (supplemented with large $|t|$ contributions that can be derived from pQCD), we estimate the statistical sensitivity of this experiment on the value of $a_{\mu}^{\mathrm{HLO}}$ to be $\sim 0.3 \%$. Such a beam is available at the CERN North Area.

\section{Detection technique}

The CERN muon beam M2 presents ideal characteristics to perform the measurement. The beam intensity is in average of about $50 \mathrm{MHz}$. The whole material budget required to reach the integrated luminosity and the required statistical precision in two years of data taking must be order of $60 \mathrm{~cm}$. In order to minimize multiple scattering and to have high radiation length the target must be of low $Z$ material. The idea is to use a segmented target of $2 \mathrm{~cm}$ thin layers, distributed in 30 identical modules. Each detection module has the length of $1 \mathrm{~m}$, a transverse surface of $10 \times 10 \mathrm{~cm}^{2}$ and consists of a target of Beryllium coupled to three stations of Silicon strip detector for tracking with high angular resolution (no magnetic field applied). Fig. 3 shows the basic layout. As tracking elements we selected the $2 \mathrm{~S}$ modules developed by the CMS collaboration for their high luminosity upgrade, read by 16 CBC binary ASICs, providing stubs (tracks segments) at $40 \mathrm{MHz}$. The hybrid transmits stubs through an optical serial line using the GBT protocol at $5 \mathrm{~Gb} / \mathrm{s}$. The estimated data throughput of the entire detector running at $40 \mathrm{MHz}$, with an average pileup of two, muons crossing the detector, is estimated to be of the order of $0.5 \mathrm{~Tb} / \mathrm{s}$. To reduce the flux we are studying a tracking trigger implemented with FPGAs. The expected signal rate is estimated to be of the order of $5 \mathrm{kHz} /$ module, amounting to $150 \mathrm{kHz}$ in total. The event signature to search for consists of a planar event with two tracks, matching in a common vertex, inside in the fiducial target volume. For particle identification we plan to exploit tracking in the downstream modules and calorimeter. They are required in order to solve the muon-electron ambiguity for electron scattering angles around(2-3) mrad $c f$. Fig. 2). Studies of the detector, performed using GEANT4, shows the intrinsic angular resolution is of $\sim 0.02 \mathrm{mrad}$. The detector acceptance covers both the region of the signal, with the electron emitted at extremely forward angles and high energies, and the so called normalization region, where the electron has much lower energy (around $1 \mathrm{GeV}$ ) and an emission angle of $30 \mathrm{mrad}$. Due to the boosted kinematics of the collisions, the detector covers most of the acceptance, and let all the scattering angles in the laboratory system to be accessed by a single detector element. 

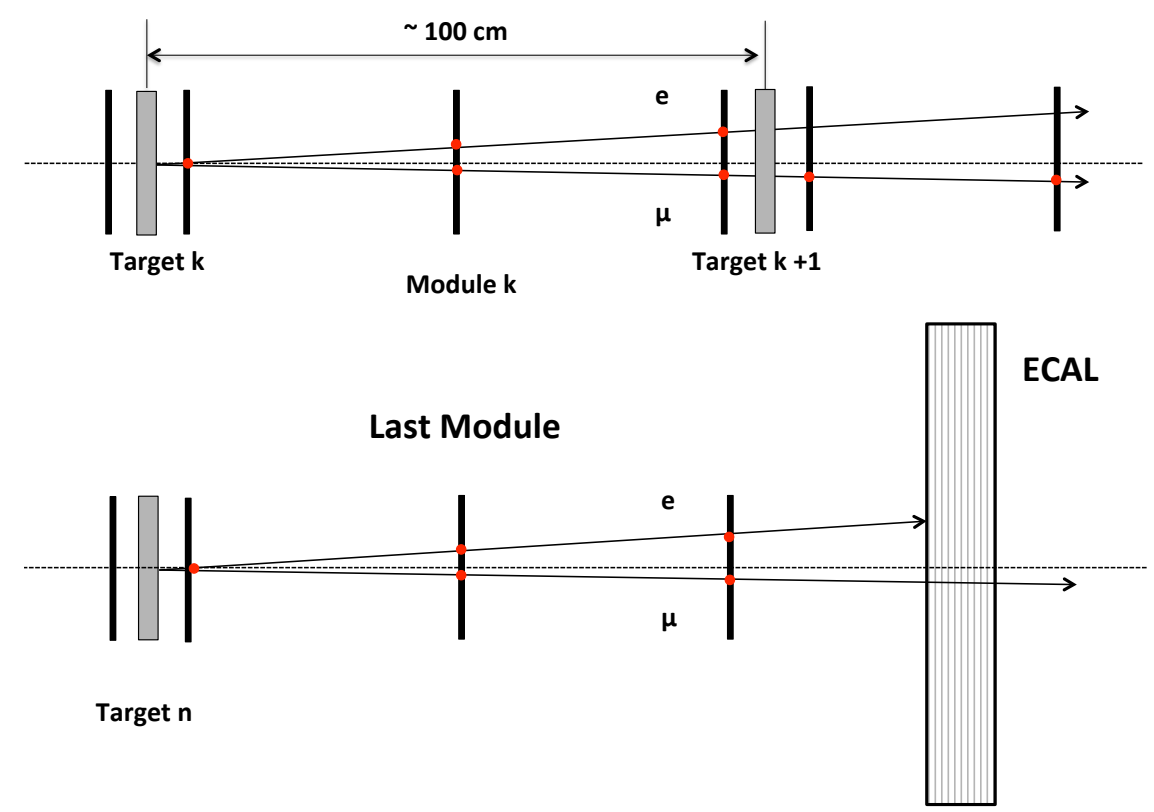

Figure 3. Scheme of a possible detector layout. (a) The detector is a modular system. Each module consists of a low- $Z$ target $(\mathrm{Be}$ or $\mathrm{C}$ ) and three silicon tracking stations within the distance of $50 \mathrm{~cm}$. (b) To perform the $\mu$-e discrimination in the case of small scattering angles (with both $\theta_{\mu}$ and $\theta_{e}$ below 5 $\mathrm{mrad}$ ) the detector is equipped with an electromagnetic calorimeter and a muon detector.

\section{Considerations on systematic uncertainties}

Significant contributions of the hadronic vacuum polarization to the $\mu e \rightarrow \mu e$ differential cross section are essentially restricted to electron scattering angles below $10 \mathrm{mrad}$, corresponding to electron energies above $10 \mathrm{GeV}$. The net effect of these contributions is to increase the cross section by a few per mille. To get a relative precision below $1 \%$ in the determination of $a_{\mu}^{\mathrm{HLO}}$ requires high statistics, a high level of control of systematic uncertainties. Goal of experiment is equivalent to a determination of the differential cross section with $\sim 10$ ppm systematic uncertainty at the peak of the integrand function ( $c f$. Fig. 1).

A crucial requirement is to keep the detection efficiency highly uniform over the entire $q^{2}$ range and over all the detector components. This motivates the choice of a purely angular measurement: an acceptance of tens of mrad can be covered with a single sensor of modern silicon detectors, positioned at a distance of about one meter from the target.

A requirement for reaching very high accuracy is to measure all the relevant contributions to systematic uncertainties from the data themselves. An important effect is the multiple scattering. It breaks the muon-electron two-body angular correlation, moving events out of the kinematic line in the 2D plot of Fig. 2. In addition, multiple scattering causes acoplanarity, while two-body events are planar, within the resolution. These facts allow effects to be modelled and measured by using data. 
Another imporant requirement is measuring very preciselly the muon maen beam energy. By reverting the two body elastic scattering kinematics we can reach the precision of few $\mathrm{MeV}$. It implies the need to control the longitudinal positions of the sensors stations to 10$100 \mu \mathrm{m}$.

We aim to control stematic effects within the experiment itself. In this respect the proposed modularity of the apparatus will help. Test with jus couple of modules planned at CERN in 2021 could provide a proof-of-concept of the proposed method.

\section{Conclusions}

The experiment MUonE presented to determine the leading hadronic contribution to the muon $g$-2, by scattering high-energy muons on atomic electrons of a low- $Z$ target through the process $\mu e \rightarrow \mu e$, is primarily based on a precise measurement of the scattering angles of the two outgoing particles as the $q^{2}$ of the muon-electron interaction can be directly determined by the electron (or muon) scattering angle. An advantage of the muon beam is the possibility of employing a modular apparatus, with the target subdivided in subsequent layers. A low- $Z$ solid target is preferred in order to provide the required event rate, limiting at the same time the effect of multiple scattering as well as of other types of muon interactions (pair production, bremsstrahlung and nuclear interactions). The normalization of the cross section is provided by the very same $\mu e \rightarrow \mu e$ process in the low- $q^{2}$ region, where the effect of the hadronic corrections on $\alpha(t)$ is negligible. Such a simple and robust technique has the potential to keep systematic effects under control, aiming at reaching a systematic uncertainty of the same order as the statistical one. For this purpose a preliminary detector layout has been described. On the theoretical side, the radiative corrections at NNLO accuracy have to be considered in full detail and a Monte Carlo event generator able to match fixed order NNLO predictions with resummation given by the Parton Shower technique is needed. By considering a beam of $150 \mathrm{GeV}$ muons with an average intensity of $\sim 1.3 \times 10^{7} \mathrm{muon} / \mathrm{s}$, currently available at the CERN North Area, a statistical uncertainty of $\sim 0.3 \%$ can be achieved on $a_{\mu}^{\mathrm{HLO}}$ in two years of data taking. A test performed using a single detector module, exploiting the muon beam facility, could provide a validation of the proposed method.

\section{References}

[1] T. Blum, A. Denig, I. Logashenko, E. de Rafael, B. Lee Roberts, T. Teubner and G. Venanzoni, arXiv:1311.2198 [hep-ph].

[2] F. Jegerlehner, EPJ Web Conf. 118 (2016) 01016;

F. Jegerlehner, Acta Phys. Polon. B 49, 1157 (2018).

[3] C. Bouchiat, L. Michel, J. Phys. Radium 22 (1961) 121;

L. Durand, Phys. Rev. 128 (1962) 441 [Erratum-ibid. 129 (1963) 2835];

M. Gourdin, E. De Rafael, Nucl. Phys. B 10 (1969) 667.

[4] C. Aubin, T. Blum, Phys. Rev. D 75 (2007) 114502;

P. Boyle, L. Del Debbio, E. Kerrane, J. Zanotti, Phys. Rev. D 85 (2012) 074504;

X. Feng, K. Jansen, M. Petschlies, D.B. Renner, Phys. Rev. Lett. 107 (2011) 081802;

M. Della Morte, B. Jager, A. Juttner, H. Wittig, JHEP 1203 (2012) 055;

T. Blum et al., Phys. Rev. Lett. 116 (2016) no.23, 232002;

B. Chakraborty, C. T. H. Davies, P. G. de Oliviera, J. Koponen and G. P. Lepage, arXiv:1601.03071 [hep-lat];

T. Blum et al. [RBC and UKQCD Collaborations], Phys. Rev. Lett. 121, no. 2, 022003 (2018); 
M. Della Morte et al., JHEP 1710, 020 (2017);

M. Krstic Marinkovic, talk at Lattice 2018, 22-28 July 2018, East Lansing, MI, USA.

[5] T. Blum, M. Hayakawa, T. Izubuchi, PoS LATTICE 2012 (2012) 022;

B. Chakraborty, C. Lehner, R. Van de Water, talks at ICHEP 2016, https://indico.cern.ch/event/432527/contributions/speakers

[6] M. Knecht, A. Nyffeler, Phys. Rev. D 65 (2002) 073034;

K. Melnikov, A. Vainshtein, Phys. Rev. D 70 (2004) 113006;

J. Prades, E. de Rafael, A. Vainshtein, arXiv:0901.0306 [hep-ph].

F. Jegerlehner, A. Nyffeler, The Muon g-2, Phys. Rept. 477 (2009) 1;

A. Nyffeler, Phys. Rev. D 79 (2009) 073012.

[7] F. Jegerlehner, A. Nyffeler, Phys. Rept. 477 (2009) 1.

[8] A. Nyffeler, arXiv:1710.09742 [hep-ph].

[9] G. W. Bennett et al. (The $g$-2 Collaboration), Phys. Rev. D73 (2006) 072003.

[10] J. Grange et al. [Muon $g$-2 Collaboration], arXiv:1501.06858 [physics.ins-det].

[11] N. Saito [J-PARC g-2/EDM Collaboration], AIP Conf. Proc. 1467 (2012) 45.

[12] G. Abbiendi, C. M. Carloni Calame, U. Marconi, C. Matteuzzi, G. Montagna, O. Nicrosini, M. Passera, F. Piccinini, R. Tenchini, L. Trentadue and G. Venanzoni, Eur. Phys. J. C (2017) 77139.

[13] C. M. Carloni Calame, M. Passera, L. Trentadue and G. Venanzoni, Phys. Lett. B 746 (2015) 325

[14] A.B. Arbuzov, D. Haidt, C. Matteuzzi, M. Paganoni and L. Trentadue, Eur. Phys. J. C 34 (2004) 267.

[15] G. Abbiendi et al. [OPAL Collaboration], Eur. Phys. J. C 45 (2006) 1.

[16] F. Jegerlehner, Springer Tracts Mod. Phys. 226, 2008.

[17] B.E. Lautrup, A. Peterman, E. de Rafael, Phys. Rept. 3 (1972) 193.

[18] S. Eidelman and F. Jegerlehner, Z. Phys. C 67 (1995) 585;

F. Jegerlehner, Nucl. Phys. Proc. Suppl. 181-182 (2008) 135

[19] S. R. Amendolia et al., Phys. Lett. B 146 (1984) 116;

S. R. Amendolia et al. [NA7 Collaboration], Nucl. Phys. B 277 (1986) 168.

[20] A.B. Arbuzov, V.S. Fadin, E.A. Kuraev, L.N. Lipatov, N.P. Merenkov, L. Trentadue, Nucl. Phys. B 485 (1997) 457;

[21] B.F.L. Ward et al., Phys. Lett. B450 (1999) 262; S. Actis et al., Eur. Phys. J. C66 (2010) 585-686.

[22] K.E. Eriksson, Nuovo Cimento 19 (1961) 1029; A.I. Nikishow, Sov. Phys. JETP (1961) 1929; K.E. Eriksson, B. Larsson and G.A. Rinander, Nuovo Cimento 30 (1963) 1434;

P. Van Nieuwenhuizen, Nucl. Phys. B 28 (1971) 429; T. V. Kukhto, N. M. Shumeiko and S. I. Timoshin, J. Phys. G 13 (1987) 725; D. Y. Bardin and L. Kalinovskaya, DESY-97230, hep-ph/9712310; N. Kaiser, J. Phys. G 37 (2010) 115005.

[23] M. Alacevich et al., work in progress.

[24] G. Balossini et al., Nucl.Phys. B758 (2006) 227-253.

[25] P. Mastrolia et al., JHEP 1711 (2017) 198.

[26] S. Di Vita et al., JHEP 1809, 016 (2018).

[27] C.M. Carloni Calame et al., JHEP 1107 (2011) 126; Nucl. Phys. Proc. Suppl. 225-227 (2012) 293

[28] M. Fael, arXiv:1808.08233 [hep-ph];

M. Fael and M. Passera, work in progress.

[29] G. Montagna et al., Nucl. Phys. B547 (1999) 39; Phys. Lett. B459 (1999) 649. 
[30] E. Derman and W.J. Marciano, Ann. Phys. 121 (1979) 147; G. D'Ambrosio, Lett. Nuovo Cim. 38 (1983) 593; J.C. Montero, V. Pleitez and M.C. Rodriguez, Phys. Rev. D58 (1998) 097505.

[31] Einstein A.,Phys. Rev. A13199912; Fermi E., Phys. Rev. A699991666.

[32] Newton I. preprint INFN 8181.

[33] Bragg B. Complete Works, Workers Playtime, edited by Unexeditor, Bologna 1997, pp. $1-10$. 